\title{
Optimizing cardiac sarcoid imaging with FDG PET: Lessons from studies of physiologic regulation of myocardial fuel substrate utilization
}

\author{
Ronald G. Schwartz, MD, MS, FACC, FAHA, ABNM, MASNC, ${ }^{a}$ and \\ Saurabh Malhotra, MD, MPH, FACC ${ }^{b}$ \\ a Division of Cardiology, Department of Medicine and Division of Nuclear Medicine, Depart- \\ ment of Imaging Sciences, University of Rochester Medical Center, Rochester, NY \\ b Division of Cardiovascular Medicine, Jacobs School of Medicine and Biomedical Sciences, \\ University at Buffalo, Buffalo, NY
}

Received Aug 27, 2018; accepted Aug 27, 2018

doi: $10.1007 / \mathrm{s} 12350-018-1438-x$

See related article, pp. 481-489

Sarcoidosis is a systemic disorder of unknown etiology, and its pathophysiology is characterized by multiorgan granulomatous inflammation and fibrosis. Cardiac sarcoidosis (CS) is associated with a worse prognosis, and diagnosis of CS is often elusive due to lack of specificity of its clinical manifestations of heart failure, conduction disease, ventricular tachycardia, and/or syncope. Imaging of CS with FDG PET CT is increasingly being utilized for clinical management of the active inflammatory process which cannot be reliably imaged by other techniques such as MRI or echocardiography prior to necrosing consequences of the disease. Successful imaging technique of the metabolic signature of CS with FDG PET CT critically exploits suppression of overwhelming myocardial glucose uptake, which is principally controlled by physiologic elevations of insulin, to allow imaging of the underlying

\footnotetext{
Electronic supplementary material The online version of this article (https://doi.org/10.1007/s12350-018-1438-x) contains supplementary material, which is available to authorized users.

Reprint requests: Ronald G. Schwartz, MD, MS, FACC, FAHA, ABNM, MASNC, Division of Cardiology, Department of Medicine and Division of Nuclear Medicine, Department of Imaging Sciences, University of Rochester Medical Center, 601 Elmwood Avenue, AC-G, Rochester, NY 14642-0001; ronald_schwartz@urmc. rochester.edu

J Nucl Cardiol 2020;27:490-3.

$1071-3581 / \$ 34.00$

Copyright (c) 2018 American Society of Nuclear Cardiology.
}

pathophysiologic intense inflammation and the associated substantial increase in glycolysis in the inflammatory cells of the infiltrative disease.

Several methods for reducing physiologic myocardial glucose uptake have been described with the aim of improving specificity for diagnosing CS, although none of them have been rigorously validated to date. In this issue of the Journal, Atterton-Evans et al report the results of a systematic review of studies comparing dietary protocols for suppression of myocardial physiologic ${ }^{18}$ F-FDG uptake. ${ }^{1}$ Based on review of studies published in MEDLINE and PubMed, the authors identified 13 studies that met their inclusion criteria, 2 of which were animal studies. Among these studies, improvement in ${ }^{18} \mathrm{~F}$-FDG uptake was either assessed qualitatively (visually) or quantitatively via assessment of standardized uptake values (SUV). Of the 11 human studies, improvement of ${ }^{18}$ F-FDG uptake was reported in 10 studies. Interestingly, in these 10 studies, all the dietary preparations that were associated with improved

${ }^{18}$ F-FDG uptake involved carbohydrate restriction, with varying degrees of fat and protein. Fasting prior to ${ }^{18} \mathrm{~F}$ FDG PET/CT varied from 4 hours to over 18 hours, but was only employed in 7 studies. The only human study in which no difference in ${ }^{18} \mathrm{~F}$-FDG uptake was observed with varying dietary preparations did not employ any carbohydrate restriction. Based on these studies, the authors conclude that fasting and low carbohydrate diet provide greater suppression of physiologic myocardial ${ }^{18}$ F-FDG uptake, and both prolonged fasting and prolonged restriction of carbohydrates will likely result in greater suppression. Their findings confirm the marked variability of dietary and fasting preparation for FDG 
PET imaging of CS in the literature and in clinical practice.

As all good scientific contributions do, this work suggests a number of questions for us to address: What is the best diet? What is the optimal time of fasting before FDG administration? Should heparin be used to help suppress myocardial glucose uptake? Given the lack of consensus, those of us involved in the day to day performance of ${ }^{18} \mathrm{~F}$-FDG PET/CT imaging for cardiac sarcoidosis may benefit from consideration of our growing understanding of physiologic regulation of myocardial fuel substrate utilization to help us optimize detection of cardiac sarcoidosis with ${ }^{18} \mathrm{~F}$-FDG PET/CT. Key concepts that can guide our thinking as we strive to suppress myocardial glucose uptake to optimize the signal to background ratio of cardiac sarcoid FDG uptake are (1) fuel substrate availability, (2) the Randle Cycle, (3) insulin resistance, (4) glycogenolysis, and (5) effects of exercise.

In 1963, Philip Randle hypothesized a competition of glucose and fatty acids driven principally by muscle and adipose tissue, which has since become known as the glucose fatty acid (Randle) cycle. ${ }^{2}$ This cycle controls fuel substrate utilization in response to supply and demand. The inhibition of glucose oxidation by fatty acids has been confirmed by many investigators, and both short- and long-term mechanisms involved in the control of glucose and fatty acid utilization at the cytoplasmic and mitochondrial level, in mammalian muscle and liver under normal and pathophysiological conditions include allosteric control, reversible phosphorylation, and the expression of key enzymes. ${ }^{3}$ Figure 1 illustrates the downstream inhibitory effects of fatty acids on glucose utilization, including inhibition of pyruvate dehydrogenase (PDH) on glycolysis produced pyruvate, and allosteric inhibition by FFA derived citrate on both the hexokinase GLUT 4 receptor and phosphofructokinase (PFK), a key regulatory step in cytosolic glucose metabolism. It remains unclear whether high concentration of fatty acids cause or result from abnormalities of carbohydrate metabolism, such as in diabetes, starvation, and Cushing syndrome. ${ }^{3}$ The interaction of glucose and fatty acids appears complex, involving glucose uptake, fatty acid oxidation, and effects of key regulatory enzymes involved in glucose and fatty acid oxidation and amino acids. Increased FFA flux participates in insulin resistance, and alleviating excess FFA has been proposed as a target for its treatment. ${ }^{4}$ Exercise and oral hypoglycemic agents stimulate stimulate glucose and fatty acid utilization. ${ }^{3}$ While skeletal muscles can store 20 minutes of glycogen, the liver holds the majority of the 1500-2000 calories of glycogen that can take 24 hours to deplete through glycogenolysis. Thus, prolonged fasting to limit fuel substrates, limiting carbohydrate intake before fasting and increasing serum free fatty acids to suppress insulin mediated glucose uptake, and avoiding exercise and oral hypoglycemic agents appear prudent interventions to suppress glucose uptake and utilization.

These observations are principally derived from whole body, hepatic, adipose and / or skeletal muscle models. Do they pertain to the heart? The heart is an omnivore: it eats what we feed it. In cardiac muscle, citrate inhibition appears more likely to affect GLUT 4 receptor rather than PFK. ${ }^{3}$ The translocation of myocardial glucose uptake facilitated by GLUT4 to the plasma membrane is stimulated by insulin, muscle contraction, and energy stress. ${ }^{3}$ In the conscious dog model, Barret demonstrated elevation of plasma insulin within the physiologic range stimulated a four-fold increase of myocardial glucose uptake using a euglycemic clamp in the absence of a change in plasma glucose or coronary blood flow. ${ }^{5}$ Importantly, FFA within the physiologic range are a major modifier of insulin-induced myocardial glucose uptake in dogs ${ }^{5}$ and in man. ${ }^{6}$ Hyperglycemia, in the absence of elevated insulin levels, has little effect on heart glucose uptake. ${ }^{5}$ Chronic diabetes induced in dogs was associated with reduced myocardial glucose extraction before and after insulin administration, and uptake of branched chain amino acids (BCAA) was stimulated by insulin. ${ }^{7,8}$ In vivo in non-diabetic conscious dogs, BCAA were the principal amino acids taken up by the myocardium at rest and during amino acid infusion. Plasma BCAA concentration and not insulin determined the rate of myocardial BCAA uptake. In addition, insulin stimulated myocardial alanine uptake. ${ }^{8}$

Our approach to myocardial sarcoid ${ }^{18}$ F-FDG PET/ CT imaging at the University of Rochester Medical Center is to incorporate these concepts of physiologic regulation of myocardial substrate uptake in our patient diet and preparation protocol (Table and Supplement), as follows:

1. We employ a regimen of low carbohydrate and high fat diet for 2 meals prior to fasting. We have reviewed our dietary recommendations with input from our dieticians and staff, and we have our scheduler and nurses review these recommendations in detail with patients prior to their procedures.

2. We allow for sufficient fasting time to deplete residual hepatic glycogen stores through glycogenolysis. This process involves an 18-hour fast for nondiabetic patients, and a 12 hours for diabetic patients to avoid hypoglycemia, which we have observed in a few diabetic patients, after 2 low carbohydrate high fat meals.

3. For patients with diabetes mellitus, we recommend administration of half-dose long acting insulin and 
Table 1. University of rochester preparation for FDG PET imaging of cardiac sarcoidosis

1. Very low carbohydrate + high fat diet for 1 day ( 2 meals) prior to fasting

2. Sufficient fasting time to deplete residual hepatic glycogen stores through glycogenolysis

(a) $18 \mathrm{~h}$ for non-diabetic patients

(b) $12 \mathrm{~h}$ for diabetic patients

3. Patients with diabetes

(a) Half-dose long acting insulin before the test

(b) Withhold short acting insulin and oral hypoglycemic agents on the morning of the test

(c) If hypoglycemia occurs, give oral glucose and avoid insulin and oral insulin sensitizers

4. Rest during the fasting period: avoid exertion and exercise

5. Supplemental heparin administration 1000 units at $45 \mathrm{~min}$ and at $15 \mathrm{~min}$ prior to FDG injection

6. Avoidance of peritoneal dialysis for 3-6 days prior to FDG injection

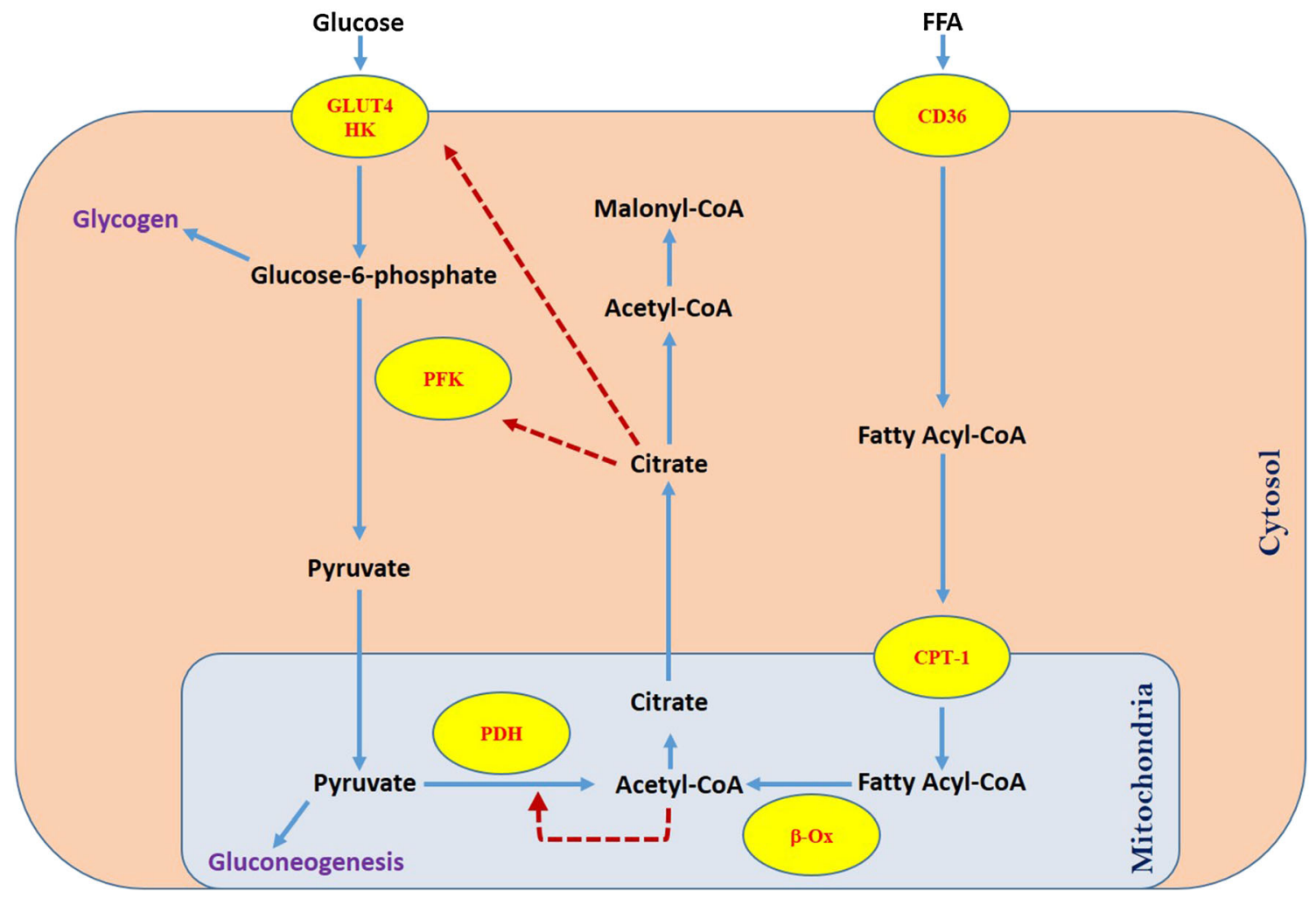

Figure 1. Mechanism of inhibition of glucose utilization by fatty acid oxidation. The extent of inhibition is graded and most severe at the level of pyruvate dehydrogenase (PDH) and less severe at the level of phosphofructokinase (PFK) and glucose uptake. PDH inhibition is caused by acetyl$\mathrm{CoA}$ and NADH accumulation resulting from fatty acid oxidation, whereas PFK inhibition results from citrate accumulation in the cytosol. The mechanism of inhibition of glucose uptake is not clear. These effects reroute glucose toward glycogen synthesis and pyruvate to gluconeogenesis. FFA, free fatty acid; GLUT4, glucose transporter 4; HK, hexokinase; CPT I, carnitine palmitoyltransferase I; $\beta$-Ox, $\beta$-oxidation. Adapted from Reference 3. 
avoidance of short acting insulin and withholding of oral hypoglycemic agents. If patients develop hypoglycemia, we use glucose but not insulin prior to ${ }^{18} \mathrm{~F}$ FDG administration, since well controlled investigations have demonstrated physiologic elevations of insulin but not glucose will drive myocardial glucose uptake. $^{5-7}$

4. We recommend our patients rest and avoid exercise during the fasting period due to lack of controlled metabolic studies of these effects on fasted patients prior to imaging.

5. To stimulate elevation of serum FFA by lipoprotein lipase cleavage of triglycerides to compete with myocardial glucose uptake, we administer heparin 1000 units $45 \mathrm{~min}$ and at $15 \mathrm{~min}$ prior to FDG injection.

6. We have discovered the need for avoidance of peritoneal dialysis for 3-6 days prior to FDG injection, which has been reported in the literature. ${ }^{9}$

The study by Atterton-Evans demonstrates the lack of consensus in the protocols reported in the literature for CS detection with ${ }^{18} \mathrm{~F}$-FDG PET/CT. The high cost of the cardiac ${ }^{18}$ F-FDG PET/CT, the serious treatment implications of false positive or false negative FDG PET studies for CS that could be caused by inadequate dietary and fasting protocols, and the inability of cardiac MRI to image much of the active inflammatory myocardial sarcoid seen by ${ }^{18} \mathrm{~F}$-FDG PET/CT suggest that strict compliance with a protocol based on the evidence of physiologic regulation of myocardial fuel substrate utilization is reasonable and prudent. Ultimately, randomized control trials will be required to document the incremental value of each component of the Rochester ${ }^{18}$ F-FDG PET/CT protocol for sarcoid imaging (Table 1). With this approach in approximately 250 cardiac PET FDG studies to date, we appear to enjoy excellent avoidance of diffuse myocardial ${ }^{18} \mathrm{~F}$ FDG uptake that compromises the accuracy of ${ }^{18}$ F-FDG $\mathrm{PET} / \mathrm{CT}$ for CS. Whether this approach which appears clinically effective optimizes sensitivity and value for detecting and monitoring the heterogenous moderate to intense ${ }^{18}$ F-FDG uptake that characterizes CS on PET $\mathrm{CT}$ imaging and management toxic immunosuppressive therapies will require further study.

\section{Disclosure}

The authors declare that they have no conflict of interest.

\section{References}

1. Atterton-Evans V, Turner J, Vivanti A, Robertson T. Variances of dietary preparation for suppression of physiological ${ }^{18} \mathrm{~F}-\mathrm{FDG}$ myocardial uptake in the presence of cardiac sarcoidosis: A systematic review. J Nucl Cardiol 2018. https://doi.org/10.1007/s1 2350-018-1379-4.

2. Randle PJ, Garland PB, Hales CN, Newsholme EA. The glucose fatty-acid cycle. Its role in insulin sensitivity and the metabolic disturbances of diabetes mellitus. Lancet 1963;1:785-9. https://doi. org/10.1016/S0140-6736(63)91500-9.

3. Hue L, Taegtmeyer H. The Randle cycle revisited: A new head for an old hat. Am J Physiol Endocrinol Metab 2009;297(3):E578-91. https://doi.org/10.1152/ajpendo.00093.2009.

4. Delarue J, Magnan C. Free fatty acids and insulin resistance. Curr Opin Clin Nutr Metab Care 2007;10:142-8. https://doi.org/10.1097/ mco.0b013e328042ba90.

5. Barrett EJ, Schwartz RG, Francis CK, Zaret BL. Regulation by insulin of myocardial glucose and fatty acid metabolism in the conscious dog. J Clin Investig 1984;74(3):1073-9. https://doi.org/ 10.1172/JCI111474.

6. Ferrannini E, Barrett EJ, Bevilacqua S, DeFronzo RA. Effects of fatty acids on glucose production and utilization in man. J Clin Investig 1983;72:1737-47.

7. Barrett EJ, Schwartz RG, Young LH, Jacob R, Zaret BL. Effect of chronic diabetes on myocardial fuel metabolism and insulin sensitivity. Diabetes 1988;37(7):943-8. https://doi.org/10.2337/dia b.37.7.943.

8. Schwartz RG, Barrett EJ, Francis CK, Jacob R, Zaret BL. Regulation of myocardial amino acid balance in the conscious dog. J Clin Investig 1985;75:1204-11.

9. Gonzalez WO, Massera D, Travin MI. False-positive 13N-ammonia/18FDG PET for evaluation of cardiac sarcoidosis in a patient on peritoneal dialysis. J Nucl Cardiol 2015;22:1323-5. 